\title{
Oromucosal/Laryngopharyngeal Solution/Spray, Solution Dosage Form
}

National Cancer Institute

\section{Source}

National Cancer Institute. Oromucosal/Laryngopharyngeal Solution/Spray, Solution

Dosage Form. NCI Thesaurus. Code C150039.

Liquid preparation consisting of a solution intended for oromucosal or laryng opharyngeal use, presented in a container with an optional spray device to allow administration as a spray. 